\title{
Clinical observation of early rehabilitation nursing on the prognosis of patients with acute myocardial infarction effect
}

\author{
Zaiying Song and Chunwen $\mathrm{Li}^{\mathrm{a}}$ \\ Beihua University, 132013, Jilin, China
}

\begin{abstract}
To explore the effect of early rehabilitation nursing on the quality of life of patients with acute myocardial infarction. Methods 41 cases of patients with acute myocardial infarction were selected according to the diagnostic criteria of acute myocardial infarction, stable vital signs, and no obvious complications. On the basis of routine nursing care, the system of early rehabilitation nursing care, the development of daily rehabilitation nursing plan. The cardiac function, clinical symptoms, quality of life and average length of stay were observed in 41 patients. Results During hospitalization, 41 patients had no deaths and all patients clinical symptoms significantly reduced; length of hospital stay was significantly decreased; heart function recovered well; the quality of life of patients significantly improved. Conclusion Early rehabilitation nursing care for patients with acute myocardial infarction is conducive to improving the condition, shorten the hospital stay, can greatly improve the quality of life of patients, it is worthy of clinical promotion.
\end{abstract}

\section{Introduction}

Cardiovascular and cerebrovascular disease is the main disease threatening the health and life of Chinese adults, including coronary heart disease and cerebral apoplexy are the cause of the Chinese people's first death. Acute myocardial infarction is a severe type of coronary heart disease (AMI), is based on coronary artery disease, coronary artery blood supply of sharp reduction or interruption of the corresponding myocardial ischemia leads to severe and persistent myocardial necrosis[1].Research shows[2], early rehabilitation nursing intervention can effectively reduce the patient's mortality rate and disability rate, improve the quality of life of patients, which is conducive to the prognosis of patients with recovery, return to society.My team of implementation of the rehabilitation nursing in 41 cases of patients with myocardial infarction during recovery period, including general nursing, activities and the rest care, diet nursing, defecation nursing, pain nursing, psychological nursing and achieved good results.

\section{Methods}

\subsection{General information}

\footnotetext{
${ }^{a}$ Corresponding author: Chunwen Li, chunwen9925@163.com
} 
41 cases of hospitalized patients from January 2014 to December in the Affiliated Hospital of Beihua University were selected and the selected of patients conforms to the standard of diagnosis of acute myocardial infarction (AMI); After the onset of 24h stable vital signs, no obvious angina pectoris without sinus tachycardia, heart failure and severe arrhythmia and cardiogenic shock, blood pressure based in the normal range. Normal body temperature and myocardial enzyme spectrum tended to turn for the better; were treated with anticoagulation, dilating coronary artery, activating blood circulation to dissipate blood stasis treatment with thrombolytic therapy for thrombolytic therapy. Male 24 cases, female 17 cases, age $(52.6 \pm 13.4)$ years old, all the patients informed consent and signed informed consent. All patients with gender, age and educational level of baseline data index, general data comparison, no statistically significant difference $(\mathrm{P}>0.05)$.

\subsection{Intervention methods}

According to the patient's condition and individual condition of the formulation and implementation of rehabilitation nursing. Rehabilitation nursing should be carried out in close monitoring, Observed the rehabilitation exercise, exercise and the end of the exercise immediately after the termination (15s) heart rate, heart rate, blood pressure changes. The following conditions were required to suspend rehabilitation activities[3].a.Heart rate $>$ resting heart rate $20 / \mathrm{min}$ or heart rate $\geq 110 / \mathrm{min}$;b.The emergence of severe arrhythmia;c. The emergence of angina, chest tightness, shortness of breath, dyspnea, palpitations, dizziness, syncope, pale, sweating and other performance;d.ST segment depression was equaled to or larger than $0.1 \mathrm{mv}$ or move up is equal to or larger than $0.2 \mathrm{mv}$; e. A significant increase in blood pressure $>200 / 100 \mathrm{mmHg}(1 \mathrm{mmhg}=0.133 \mathrm{kPa})$ or systolic blood pressure than resting levels rise was more than or equal to $30 \mathrm{mmHg}$ or decline was more than or equal to $10 \mathrm{mmHg}$; f.The subjective fatigue (RPE was more than or equal to level 14), after exercise6 8min breathing, heart rate did not returned to pre exercise state, or cause insomnia, fatigue,edema[4];g.The heart rate was not even speeding up but slow down with the increase of load, should stop or go back to the last stage of exercise[5].

3 Patients of intravenous thrombolysis after $24 \mathrm{~h}$ were given rehabilitation nursing, by the professional training of nurse was responsible for guiding the implementation of local departments to develop acute myocardial infarction early rehabilitation training program[6].See Table 1

Table 1. Rehabilitation content

\begin{tabular}{|l|l|}
\hline Time (d) & Activity content and rehabilitation content \\
\hline 1 & $\begin{array}{l}\text { Bed rest, in the heart of the passive stand up, 2 times a day, each time } 20 \text { min. } \\
\text { Activly participate in physical activity, and the introduction of cardiac rehabilitation } \\
\text { program and the ward environment, maintain emotional stability of patients. }\end{array}$ \\
\hline $2-3$ & $\begin{array}{l}\text { Semi supine or sitting position, active physical activity } 2 \text { times, each time 5-10min. } \\
\text { Assist the bed wash, eat, use the potty bed. Introduce the advantages of early } \\
\text { rehabilitation nursing, the attention items of rest and diet,obtain trust. }\end{array}$ \\
\hline $4-5$ & $\begin{array}{l}\text { Sit and stand by the bed chair } 2 / \text { d, } 10 \text { min each time, sitting face and diet. Explain } \\
\text { the principles of the treatment of myocardial infarction and rehabilitation procedures, } \\
\text { allowing reading newspapers, listening to music and visiting. }\end{array}$ \\
\hline $6-8$ & $\begin{array}{l}\text { Stand, walk freely walking indoor toilet, eating, Explain the importance of drug } \\
\text { treatment and adherence to medication }\end{array}$ \\
\hline
\end{tabular}




\begin{tabular}{|l|l|}
\hline $9-10$ & $\begin{array}{l}\text { Indoor activities increased, the basic living. To explain the knowledge of emergency } \\
\text { treatment of myocardial infarction. }\end{array}$ \\
\hline $11-12$ & $\begin{array}{l}\text { Outdoor corridor slow line, each time } 50-100 \mathrm{~m}, 2 \text { times } / \mathrm{d} \text {. The relationship between } \\
\text { exercise and rest. }\end{array}$ \\
\hline $13-14$ & Up and down the stairs movement, before the discharge guidance, regular follow-up. \\
\hline
\end{tabular}

\subsection{Diet Care Nursing}

The nursing staff assisted in the arrangement of the rehabilitation period of patients. Guidancing for patients to eat should not be too full, eat less and eat more time. The food was easy to digest with less fat and less gas production was appropriate, limiting the amount of sodium intake. If the serum lipid levels were still significantly abnormal, according to the characteristics of dyslipidemia patients, choosing appropriate lipid-lowering drugs[7].

\subsection{Care of patients with disorders of urination and defecation}

Constipation is common in patients with AMI, should be within 3 days of defecation in bed, do not forcibly defecate, explain to the patient in bed defecation had great significance to control the disease, not because of fear of defecate on the bed and can not eat, it would increase the risk of constipation. At the same time, from the first day of hospitalization, the daily morning honey $20 \mathrm{ml}$ plus $80 \mathrm{ml}$ temperature with boiling water to drink, abdominal massage (clockwise) $2 / \mathrm{d}$, in order to promote intestinal peristalsis.

\subsection{Pain nursing}

Myocardial infarction patients often have severe chest pain, nursing staff should follow the doctor's advice given nitroglycerin, morphine and other drugs to prevent pain, causing shock and arrhythmia, and inquired the pain and concomitant the changes of in symptoms, to monitor the change of blood pressure in patients with attention, and attended to patients with no pulse acceleration, respiratory depression and other adverse reactions.

\subsection{Mental nursing}

Patients with severe chest pain should try to keep a nurse accompanied by the patient side.Because of the particularity of myocardial infarction disease, patients may produce negative emotions. The domestic and foreign studies had found that AMI was prevalent in patients with depression, anxiety, fear, pessimism, despair and other psychological. Had a bad mood would affect the treatment and rehabilitation of disease[8]. Rozanski A[9] found that there was significant correlation between the lack of emotional support and myocardial infarction mortality. The medical staff should comfort the patients and explain the bad mood would increase the heart load and myocardial oxygen consumption, which was not conducive to disease control. The nurse with the doctors rescue at the same time, should do the work to appease the patients and the families of patients[10]. 


\section{Evaluation index}

Observed in 41 patients in treadmill exercise, hospitalization duration, self-care ability etc. And observed in 41 cases of CQQC (quality of life questionnaire Chinese cardiovascular disease score). The CQQC questionnaire was written by Jiangsheng L, Chinese Rehabilitation Medical Association cardiovascular disease Specialized Committee, and cardiovascular rehabilitation medical journal. According to the definition of the quality of life of the WHO, to develop scale according to three aspects as physiological, psychological and social conditions of our country, and the appropriate reference the questionnaire[11], is divided into physical, disease , medical conditions , general life , social psychological status, working conditions and other 6 projects, 24 items, for respondents according to their own situation of the corresponding answer, answer according to the score.

\section{Conclusions}

After 2 weeks, 41 cases of patients with the average treadmill walking distance, average hospitalization days, the recovery of heart function. See Table 2.

Table 2. Effect Analysis

\begin{tabular}{|c|c|c|c|c|}
\hline \multirow{2}{*}{ Gender } & \multirow{2}{*}{$\begin{array}{l}\text { Average walking } \\
\text { distance (m/ 10 min) }\end{array}$} & \multirow{2}{*}{$\begin{array}{l}\text { Length of stay } \\
\text { (days) }\end{array}$} & \multicolumn{2}{|l|}{ Heart function classification (n) } \\
\cline { 3 - 5 } & $326 \pm 140$ & $18.9 \pm 4.4$ & I $\sim$ II & III $\sim$ IV \\
\hline male & $347 \pm 120$ & $18.5 \pm 4.2$ & 13 & 4 \\
\hline Female & &
\end{tabular}

After the intervention, the CQQC score of all patients with early rehabilitation nursing was good, and the overall effect was good. Patients reflected the good recovery, quality of life was significantly improved. See Table 3.

Table 3. Patients' quality of life score

\begin{tabular}{|c|c|c|}
\hline Gender & Male $(24$ cases $)$ & Female $(17$ cases $)$ \\
\hline Physical condition & $17.24 \pm 6.93$ & $16.34 \pm 3.73$ \\
\hline The illness & $16.23 \pm 3.98$ & $16.83 \pm 2.54$ \\
\hline Medical condition & $5.75 \pm 0.63$ & $5.29 \pm 0.84$ \\
\hline General life & $10.02 \pm 2.89$ & $6.53 \pm 1.72$ \\
\hline Working condition & $6.08 \pm 1.07$ & $6.67 \pm 1.57$ \\
\hline
\end{tabular}

\section{Discussion}

Analysis of the effect of early rehabilitation nursing on patients with acute myocardial infarction Table 2 shows, the length of hospital stay was significantly shorter, indicating that no adverse effect of early rehabilitation nursing on patients with acute myocardial infarction, to resume working ability of patients and improve the prognosis of patients, and reduce the cost of hospitalization, reduce the burden of family and society has an important significance. It is safe and feasible. The purpose of early rehabilitation nursing is to strive for patients' life as early as possible and early discharge, and to monitor the activities of the transition to go home under the no monitoring of the security activities[12]. This stage of activity is to "not too much," will not cause a infarction or even death as the basic principles. But in the early phase of myocardial infarction, many factors affect the oxygen consumption of patients, the movement itself on patients with acute myocardial infarction (AMI) existence certain risk, the effects of various exercise on heart rate and blood pressure is inconsistent, inappropriate exercise can increase the myocardial oxygen consumption and aggravate myocardial damage, induced myocardial infarction and heart failure[12].Therefore, nursers in rehabilitation of 
patients with acute myocardial infarction (AMI) to evaluation of patients with personality and social psychological factors, must be fully taken into account and to minimize the adverse impact of these movements.

The research results indicate that early rehabilitation nursing patients quality of life scores higher after nursing. The study shows that the rehabilitation nursing measures is conducive to promoting the improvement of the patient's condition and the quality of life of patients[13-14].The study [15]found that the mortality and disability rate were higher in patients with acute myocardial infarction, but the early rehabilitation nursing measures could improve the quality of life of patients to a certain extent in the early rehabilitation nursing measures. Because of myocardial infarction patients with long-term bed is very easy to cause deep vein thrombosis, reduce exercise capacity, resulting in waste of the syndrome, and is not conducive to the recovery of heart function[16]. Early rehabilitation nursing by functional exercise guidance on patients, promote the patient recover as soon as possible, it can reduce caused by prolonged bed rest complications such as angina, arrhythmia and heart failure and other, eliminate fatigue of patients, improve patient self-care ability and athletic ability, and greatly ease the patient's mental burden[17]. There is conducive to the patient's condition improved[18]. And patients through early rehabilitation guidance, improve the treatment of active and active, to overcome their own bad mood, enhance the confidence to overcome the disease.

In summary, the use of early rehabilitation nursing is of very important significance in patients with acute myocardial infarction, can greatly improve the quality of life of patients, improve the patients' satisfaction degree of nursing work, is to improve the patient's condition, it is worth clinical promotion.

\section{References}

1. You LM, Sun GZ, Yuan L. People's Health Publishing House. Department of internal medicine nursing school, Third edition, Beijing.M.164-170(2002).

2. Dong ZQ. Nursing practice and research. Early rehabilitation exercise and nursing in patients with acute myocardial infarction. J. 8 (8),52 -53(2011).

3. Liu JS, Dai RZ, Cheng YZ. Journal of cardiovascular rehabilitation medicine. Percutaneous coronary artery interventional therapy after rehabilitation program.J.15 (2),125-129(2006).

4. Zhen YJ, Wang Y, Liu SY. Journal of Hebei Medical University. In the early stage of acute myocardial infarction cardiac rehabilitation.J.32 (10),1230-1232(2011).

5. Zhou SY, Zhang ZY, Tang CL, et al. Journal of modern nursing. The influence of early rehabilitation nursing on the complications after interventional therapy for acute myocardial infarction.J.15 (20),1938-1939(2009).

6. Su Q, Li J, Sun HP. China's recovery. Early rehabilitation nursing of patients with acute myocardial infarction after intravenous thrombolysis.J.1(4),283-284(2006).

7. Hu DY .Chinese Journal of Department of Internal Medicine. Chinese expert consensus on thrombolytic therapy for acute ST segment elevation myocardial infarction.J.47(2),170-174(2008).

8. L Wi, Zhou CH, Gao DS. Chinese Journal of Behavioral Medical Science. The psychological state in patients with acute myocardial infarction and complications related to correlation.J.11 (3),265267(2002).

9. Zhang SH. China Medical Herald. Clinical nursing pathway in patients with acute myocardial infarction (AMI) application.J.8 (2): 1-17. (35),115-116(2011).

10. Xie H, Dai HQ. Mental illness and mental health.SCL-90 scale evaluation.J.6 (2),156-159(2006).

11. Dong ZQ. Nursing practice and research. Early rehabilitation exercise and nursing in patients with acute myocardial infarction.J.8(8),52-53(2011).

12. Li DM, Li JS, R WS, et al. International nursing journal. Clinical nursing pathway in acute myocardial infarction patients with pre hospital first aid in application.J.31 (10),1848-1850(2012).

13. Xiong LL. Chinese cosmetic medicine. Research progress of clinical nursing pathway in elderly patients with acute myocardial infarction.J.19 (Z3),78-79(2010). 
14. Liu Q. Nursing research. Evaluation of application effect of clinical nursing pathway in patients with acute myocardial infarction.J.24 (29),2691-2692(2010).

15. Apóstolo JL, Kolcaba K. Arch Psychiatr Nurs. The effects of guided imagery on comfort depression anxiety, and stress of psychiatric inpatients with depressive disorders.J.23(6),403411(2009).

16. Weda DT, Collin C.Int Disabil Stud. The Barthel ADL Index: a standard measure of physical disability.J.10(2),64-67(1988).

17. Guo PQ, Wang CY, Yang QY, et al. Journal of Chinese medical science. Application of early rehabilitation nursing in patients with acute myocardial infarction.J.9(35),8601-8602(2009).

18. Zhao ZH, Zhang SQ, Sun HP. China Rehabilitation. Early rehabilitation nursing care for patients with acute myocardial infarction after intravenous thrombolysis in patients with recovery and complications.J.24 (4),277-278(2009). 